\title{
MECHANICAL PROPERTIES OF NORMAL AND OSTEOARTHRITIC HUMAN ARTICULAR CARTILAGE
}

\author{
Dale L. Robinson ${ }^{\mathrm{a}}$, Mariana E. Kersh ${ }^{\mathrm{a}, \mathrm{b}}$, Nicole C. Walsh ${ }^{\mathrm{c}, 1}$, David C. Ackland ${ }^{\mathrm{a}}$, \\ Richard N. de Steiger ${ }^{\mathrm{d}, \mathrm{e}}$, Marcus G. Pandy ${ }^{\mathrm{a}}$
}

aDept of Mechanical Engineering, University of Melbourne, Parkville, Victoria 3010, Australia

${ }^{\mathrm{b}}$ Dept of Mechanical Science and Engineering, University of Illinois at Urbana-Champaign, Illinois 61801, USA

'St Vincent's Institute of Medical Research and Department of Medicine at St Vincent's

Hospital, University of Melbourne, Parkville, Victoria 3010, Australia

${ }^{\mathrm{d} D e p t}$ of Surgery, University of Melbourne, Parkville, Victoria 3010, Australia

${ }^{\text {eDept }}$ of Surgery, Epworth Healthcare, Melbourne, Victoria 3010, Australia

Submitted as an original article to Journal of the Mechanical Behavior of Biomedical Materials Word count: Text: 6,261 (Introduction to Discussion), Abstract: 295.

08 January 2016

Corresponding author:

Dale L. Robinson

Department of Mechanical Engineering

University of Melbourne, Victoria 3010, Australia

Email: drobinson@unimelb.edu.au

Phone: +61383440405

Fax: +6138344 4290

${ }^{1}$ Current address: Murdoch Childrens Research Institute, Parkville, Victoria 3052, Australia 


\section{Abstract}

Isotropic hyperelastic models have been used to determine the material properties of normal human cartilage, but there remains an incomplete understanding of how these properties may be altered by osteoarthritis. The aims of this study were to (1) measure the material constants of normal and osteoarthritic human knee cartilage using isotropic hyperelastic models; (2) determine whether the material constants correlate with histological measures of structure and/or cartilage tissue damage; and (3) quantify the abilities of two common isotropic hyperelastic material models, the neo-Hookean and Yeoh models, to describe articular cartilage contact force, area, and pressure. Small osteochondral specimens of normal and osteoarthritic condition were retrieved from human cadaveric knees and from the knees of patients undergoing total knee arthroplasty and tested in unconfined compression at loading rates and large strains representative of weight-bearing activity. Articular surface contact area and lateral deformation were measured concurrently and specimen-specific finite element models then were used to determine the hyperelastic material constants. Structural parameters were measured using histological techniques while the severity of cartilage damage was quantified using the OARSI grading scale. The hyperelastic material constants correlated significantly with OARSI grade, indicating that the mechanical properties of cartilage for large strains change with tissue damage. The measurements of contact area described anisotropy of the tissue constituting the superficial zone. The Yeoh model described contact force and pressure more accurately than the neoHookean model, whereas both models under-predicted contact area and poorly described the anisotropy of cartilage within the superficial zone. These results identify the limits by which isotropic hyperelastic material models may be used to describe cartilage contact variables. This study provides novel data for the mechanical properties of normal and osteoarthritic human 
articular cartilage and enhances our ability to model this tissue using simple isotropic hyperelastic materials.

Keywords: Hyperelastic, neo-Hookean, Yeoh, incompressible, large deformation, radial strain 


\section{Introduction}

Articular cartilage is a thin layer of soft tissue that lines the ends of bones in diarthrodial joints. It provides the contact surfaces for load transfer between bones and facilitates near-frictionless joint articulation (Ateshian and Mow 2005). Cartilage tissue achieves these functions through its complex structure comprised of a depth-dependent collagen fibril network and proteoglycans (PGs) saturated with an interstitial fluid phase. Degeneration of cartilage due to osteoarthritis (OA) alters its internal structure, leading to changes in its mechanical properties (Setton et al., 1999). Accurate knowledge of these mechanical changes could facilitate a better understanding of the initiation and progression of OA and lead to improved treatment therapies.

Cartilage undergoes large finite deformations in vivo with compressive strains of up to $30 \%$ (Bingham et al., 2008), and its compressive stiffness is characterized by a nonlinear stress-strain curve that varies with strain rate (Oloyede et al., 1992). To simulate physiological loading, cartilage has been loaded at strain rates greater than 15\%/s (Henak et al., 2014; Park et al., 2004; Pierce et al., 2009). This produces an 'instantaneous' response of the tissue, where there is negligible interstitial fluid-flow so that the cartilage behaves as an incompressible elastic solid (Ateshian et al., 2007; Pierce et al., 2009). Hyperelastic materials may be used to model cartilage tissue because their strain energy functions not only represent the nonlinear stress-strain relationships of cartilage but also describe its incompressible behavior (Henak et al., 2014). However, few data are available for the hyperelastic material properties of healthy human cartilage (Anderson et al., 2008; Henak et al., 2014; Pierce et al., 2009), and no previous studies to our knowledge have used these models to determine the material constants of osteoarthritic human cartilage. 
Previous work has shown that for linear-elastic, biphasic or hyperelastic models of cartilage, the respective material constants vary considerably across joints and between individuals (Armstrong and Mow 1982; Henak et al., 2014; Pierce et al., 2009; Shepherd and Seedhom 1999). The variations in the material constants have been found to correlate with tissue composition (Kiviranta et al., 2006; Rieppo et al., 2003) and/or OA severity (Armstrong and Mow 1982; Kiviranta et al., 2008; Kleemann et al., 2005; Saarakkala et al., 2003). Whilst the material constants in hyperelastic cartilage models are most likely to change due to OA, the extent of these changes and whether they can be predicted by variations in tissue structure or degree of OA severity remain unknown.

Isotropic material models have been used to describe cartilage in finite element models of entire joints because they are readily implementable and have low computational cost (Haut Donahue et al., 2003; Kiapour et al., 2014). The ability of isotropic hyperelastic models to predict contact mechanics for macroscopically undamaged cartilage was recently evaluated by Henak et al. (2014). They found that the neo-Hookean and Veronda Westmann hyperelastic models both yielded an RMS error in predicted contact pressure of approximately $24 \%$, and concluded that contact area and pressure predictions at the whole-joint level are relatively insensitive to the type of constitutive model used. However, these authors also calculated contact pressure using measurements of contact force obtained for small, idealized half cylinders of cartilage and found clear differences between the neo-Hookean and Veronda Westmann models. Direct measurements of contact area and the use of models that incorporate specimen-specific geometry may provide a more accurate means of analyzing hyperelastic model predictions at smaller length scales and help to address the differences in predicted contact mechanics (e.g., contact area and pressure) derived from small- and large-scale geometries. 
Therefore, the aims of the present study were to:

(1) Measure the hyperelastic material constants of normal and osteoarthritic human knee cartilage using isotropic hyperelastic models;

(2) Determine whether the hyperelastic material constants correlate with structural parameters and/or severity of cartilage damage; and

(3) Determine the accuracy with which isotropic hyperelastic models that incorporate specimen-specific geometry describe measurements of contact force, contact area, average contact pressure and anisotropy of the superficial zone.

We hypothesized that the hyperelastic material constants change with damage to the tissue and that these changes correlate with the structural parameters of cartilage.

\section{Methods}

\subsection{Specimen preparation}

Fifty-one cylindrical osteochondral plugs (each $5 \mathrm{~mm}$ in diameter) were harvested from the cartilage and bone removed from the tibiofemoral joints of 10 patients ( 4 male, 6 female, age 69.7 \pm 9.3 years) undergoing total knee arthroplasty (TKA). Femoral specimens were harvested from the medial and lateral femoral condyles. Tibial specimens were harvested from the medial and lateral compartments of the tibial plateau in regions that were covered or uncovered by the menisci. An experienced orthopaedic surgeon (RdeS) confirmed upon visual inspection that the cartilage harvested from the knees of these patients exhibited typical defects associated with OA, such as fibrillation, erosion and cracking. The cartilage-bone interface was left in place to maintain the natural boundary condition of the cartilage during testing (Morel and Quinn 2004). 
Forty-three additional cylindrical osteochondral plugs (each $5 \mathrm{~mm}$ in diameter) were harvested from the tibiofemoral joints of three human knee cadavers ( 1 male, 2 female, age 59.1 $1 \pm 7.2$ years). These specimens were obtained for the purpose of testing normal or minimally-damaged cartilage tissue. Arthroscopic examination was performed on each cadaver knee by the same orthopaedic surgeon to verify that the cartilage was free of macroscopic defects. Approval for this study was obtained from the Ethics Committees of the University of Melbourne and the Epworth Hospital in Melbourne (University of Melbourne ethics IDs 1341081 and 1238067 and Epworth Healthcare ethics ID 54412).

Each $5 \mathrm{~mm}$ cylindrical osteochondral plug was harvested from either the medial or lateral compartment of the tibiofemoral joint using a hand-held coring tool (Fig. 1). The thin layer of subchondral bone was leveled parallel to the articular surface using a diamond saw (Buehler, Lake Bluff, IL). The inner $3 \mathrm{~mm}$ diameter core was extracted with a dermal punch for mechanical testing (Fig. 1b, c) while the outer ring was fixed and prepared for histological analysis (see below). Each $3 \mathrm{~mm}$ cylindrical plug was immersed in phosphate-buffered saline (PBS) with protease inhibitors and stored at $4^{\circ} \mathrm{C}$. Before testing, specimens were allowed to equilibrate in this solution at room temperature $\left(22^{\circ} \mathrm{C}\right)$ for $30 \mathrm{~min}$ to achieve swelling equilibrium. The specimens sourced from the TKA surgeries were tested 19.4 \pm 7.2 hours postsurgery and did not undergo a freeze-thaw cycle. The specimens obtained from the cadavers were tested $28.0 \pm 16.8$ hours following joint dissection. Twenty of the cadaver specimens underwent a single freeze-thaw cycle, a procedure which has been shown to have little effect on the mechanical properties of cartilage (Changoor et al., 2010; Kiefer et al., 1989; Salai et al., 1997; Szarko et al., 2010). 


\subsection{Geometry acquisition and measurements}

The surface geometry of each $3 \mathrm{~mm}$ osteochondral plug was acquired prior to mechanical testing using an optical reconstruction method (Fig. 2). The plug was mounted on a turnstile and photographed by a charge-coupled device (CCD) camera (GC1020C, Prosilica, Stadtroda, Germany) as it rotated in $5^{\circ}$ increments from 0 to $355^{\circ}$ (Fig. 2a). In each image, the edges of the plug were segmented using Amira (FEI, Hillsboro, OR) and then aligned to a global coordinate system (Fig. 2b, c). The resulting point cloud was wrapped and surfaced with Geomagic Studio (v 10, Geomagic, Bethesda, MD) (Fig. 2e). The visible boundary of the tidemark was similarly segmented and used to create an interpolated surface representing the interface between the articular cartilage and calcified cartilage (Fig. 2d). The geometry of the combined layer of subchondral bone and calcified cartilage was approximated by vertically extruding the tidemark to the surface of the turnstile. To validate the geometry acquisition method, a $3 \mathrm{~mm}$ diameter cylinder of vinyl rubber and epoxy laminate was reconstructed and compared to the geometry acquired using a micro-computed tomography (CT) scanner (resolution $14 \mu \mathrm{m}$, vivaCT 40, Scanco Medical, Bruttisellen Switzerland). The surfaces of the reconstructed rubber and rubber/epoxy interface differed from the CT data by $0.04 \pm 0.03 \mathrm{~mm}$ and $0.05 \pm 0.04 \mathrm{~mm}$, respectively, consistent with results obtained previously by others (Haut et al., 1998). Relative to the size of each specimen, differences on this order were assumed to be negligible.

Cartilage volume $\left(\mathrm{V}_{\text {cart }}\right.$ ) was calculated from the point cloud data using Matlab (Mathworks, Natick, MA). The specimen diameter ( $\left.\mathrm{d}_{\text {cart }}\right)$ was calculated by averaging the diameters of circles fit to the point cloud in $0.01 \mathrm{~mm}$ axial increments. Cartilage thickness $\left(\mathrm{t}_{\text {cart }}\right)$ was approximated as the height of a uniform cylinder with a diameter of $d_{\text {cart }}$ that yielded a volume of $V_{\text {cart. Ten }}$ specimens with $t_{c a r t} / d_{c a r t}$ greater than 1.0 were excluded from the analysis due to the potentially 
negative effects of bending during testing. The remaining specimens $(n=84)$ were considered in all subsequent analyses.

\subsection{Mechanical testing}

Each $3 \mathrm{~mm}$ cylindrical plug was loaded in unconfined compression using a custom loading apparatus (Fig. 3). The apparatus consisted of a central loading column translated by a linear actuator (404XR, Parker Hannifin, Cleveland, OH) instrumented with a load cell (LPU-100, Transducer Techniques, Temecula, CA) and a linear variable differential transformer (Model S5 HN AC-AC, Honeywell, Morristown, NJ). A stainless steel rod mounted at the end of the loading column and a glass plate clamped beneath the rod constituted the upper and lower loading platens, respectively.

Prior to testing, the loading apparatus was calibrated by performing a series of unconfined compression experiments on three cylindrical rubber specimens (each of diameter $3 \mathrm{~mm}$ and height $3 \mathrm{~mm}$ ). Each rubber specimen was subjected to a loading rate of $20 \% / \mathrm{sec}$ until $30 \%$ axial engineering strain was reached, and these tests were then repeated using a calibrated microtesting device (Model 5548 microtester, Instron, Norwood, MA). The root-mean-squared (RMS) difference in the contact force measured by the loading apparatus and the microtester, averaged across the three specimens, was $\pm 0.66 \%$ of the peak contact force $(51.2 \mathrm{~N})$.

The bony side of each plug was fixed to the stainless-steel rod using cyanoacrylate (Athanasiou et al., 1994) and the articular surface was then pressed against a glass plate. A thin film of PBS was applied to the glass plate to reduce friction between the articular surface and the glass. An inverted microscope (Axiovert 40 MAT, Carl Zeiss, Oberkochen, Germany) coupled with a digital camera (LW230C, Lumenera, Ottawa, Canada) was positioned under the glass plate to 
measure the contact area of the articular surface during loading. A side-mounted CCD camera (GC1020C, Prosilica, Stadtroda, Germany) was used to image the lateral deformation of the specimen during loading (Fig. 3). The digital camera and CCD camera sampled at 12 frames/sec and 33 frames/sec, respectively.

Each osteochondral specimen was slowly lowered toward the glass plate until a small, detectable contact force was recorded $(<0.3 \mathrm{~N})$. Compression was then applied at a strain rate of $20 \% / \mathrm{sec}$ up to a maximum of $30 \%$ axial engineering strain, calculated using $t_{\text {cart }}$ as the reference. These values of loading rate and strain were based on estimates reported for walking (Liu et al., 2010) and are similar to the values used by others (Henak et al., 2014).

\subsection{Axial strain of the combined layer of subchondral bone and calcified cartilage}

Using the images acquired by the side-mounted CCD camera, the combined layer of subchondral bone and calcified cartilage $(\mathrm{SB}+\mathrm{CC})$ was segmented along the left and right edges at each time point using ImageJ (National Institutes of Health, Bethesda, MD) (Fig. 4a, b). Differences in lateral deformation, intensity and color were used to distinguish the interface between cartilage and calcified cartilage. The axial engineering strains on the left and right edges, $\varepsilon_{\mathrm{L}}$ and $\varepsilon_{\mathrm{R}}$, respectively, were calculated at each time increment as follows:

$$
\begin{gathered}
\varepsilon_{L}=\frac{\Delta t_{L}}{t_{L}}, \\
\varepsilon_{R}=\frac{\Delta t_{R}}{t_{R}}
\end{gathered}
$$

where $t_{L}$ and $t_{R}$ are the thicknesses of the $S B+C C$ on the left and right edges, respectively (Fig. $4 a, b)$. 


\subsection{Contact variables}

Contact area was quantified at each time point of the compression by segmenting and fitting an ellipse to the image acquired from the inverted microscope. The semi-minor and semi-major diameters $\left(d_{y}\right.$ and $d_{z}$, respectively; Fig. $\left.4 c, d\right)$ were used to calculate the minimum and maximum radial strains as follows:

$$
\begin{aligned}
& \varepsilon_{y}=\frac{\Delta d_{y}}{d_{y}}, \\
& \varepsilon_{z}=\frac{\Delta d_{z}}{d_{z}} .
\end{aligned}
$$

The radial strain ratio $\left(\varepsilon_{z} / \varepsilon_{y}\right)$ was taken as a measure of anisotropy of the superficial zone of the cartilage (Demarteau et al., 2006). Average contact pressure was found by dividing the contact force by the corresponding contact area measured at each time point.

\subsection{Histological Analysis}

The structure and composition of the outer ring of cartilage and bone (outer diameter $5 \mathrm{~mm}$; wall thickness $1 \mathrm{~mm}$ ) was analyzed by histology. The material properties of the outer ring were assumed to be consistent with the inner $3 \mathrm{~mm}$ diameter osteochondral plug. Each ring was fixed in $10 \%$ neutral buffered formalin for $24 \mathrm{~h}$, decalcified and embedded in paraffin, and finally sectioned into $5 \mu \mathrm{m}$ slices (see Fig. 1b, d). Sections were stained with safranin-orange/fast green (SFG) to assess PG content (Rosenberg 1971) and with picrosirius red (PSR) to visualize the collagen fibril network and enable measurement of the thicknesses of the depth-dependent zones.

To evaluate indicative PG content, the relative PG thickness was calculated in the SFG sections by segmenting and calculating the average thickness of the safranin-orange stained region using ImageJ, and then normalizing by $t_{\text {cart }}$ (Fig. 5a). The SFG sections were graded independently for 
cartilage damage, a measure of OA severity, by two blinded investigators (DLR, NCW) using the OARSI grading system (Pritzker et al., 2006), and the data then averaged for each specimen to yield the final OARSI grade (Fig. 5b-f). Inter-observer agreement was determined by Cohen's kappa coefficient calculated for the two sets of results.

The PSR sections were imaged using polarized light microscopy, which allows visualization of the collagen fibril network (Bi et al., 2005). Using this method, regions with highly organized collagen fibrils, typical of the superficial and deep zones (SZ and DZ), appear bright red to yellow in color, whereas regions with less collagen organization, characteristic of the intermediate zone (IZ), are much darker. Based on these colors and pixel intensities, each section was segmented into three zones (SZ, IZ, and DZ) using ImageJ (Fig. 6). The zonal thicknesses were measured and then normalized by $\mathrm{t}_{\text {cart }}$ to obtain the normalized thicknesses of the superficial, intermediate, and deep zones, $\mathrm{t}_{\mathrm{SZ}}, \mathrm{t}_{\mathrm{IZ}}$, and $\mathrm{t}_{\mathrm{DZ}}$, respectively.

\subsection{Modeling of cartilage and the layer of $\mathrm{SB}+\mathrm{CC}$}

Two isotropic hyperelastic constitutive models, the neo-Hookean and Yeoh models, were used to represent cartilage. In both models, cartilage was assumed to be incompressible due to the high loading rate used in the experiments (Henak et al., 2014). The neo-Hookean constitutive model has a strain energy function described by:

$$
W=\frac{1}{2} \mu\left(\bar{I}_{1}-3\right)+\frac{1}{2} K_{0}\left(J^{e l}-1\right)^{2}
$$

where $\mu$ is the shear modulus under infinitesimal strain, $\bar{I}_{1}$ is the first deviatoric invariant, which is a function of the principal strains, $\mathrm{K}_{0}$ is the initial bulk modulus, and $\mathrm{J}^{\mathrm{el}}$ is the elastic volume 
ratio. For incompressible behavior, the second term vanishes. The Yeoh constitutive model has a strain energy function of the form:

$W=C_{10}\left(\bar{I}_{1}-3\right)+C_{20}\left(\bar{I}_{1}-3\right)^{2}+C_{30}\left(\bar{I}_{1}-3\right)^{3}+\frac{1}{D_{1}}\left(J^{e l}-1\right)^{2}+\frac{1}{D_{2}}\left(J^{e l}-1\right)^{4}+\frac{1}{D_{3}}\left(J^{e l}-1\right)^{6}$,

where $\mathrm{C}_{10}, \mathrm{C}_{20}, \mathrm{C}_{30}, \mathrm{D}_{1}, \mathrm{D}_{2}$ and $\mathrm{D}_{3}$ are independent material constants. For incompressible material behavior, the last three terms vanish. In the present study, $\mathrm{C}_{30}$ was assumed to be zero because a second-order strain energy function was sufficient to describe quadratic stress-strain relationships, a trend observed in previous studies when cartilage was compressed at high strain rates (Henak et al., 2014; Oloyede et al., 1992). The SB+CC was modeled as a linear-elastic solid with a Young's modulus of ESB+CC (obtained from experiment) and a Poisson's ratio of 0.35, which is identical with the value reported for cortical bone (Ashman et al., 1984).

\subsection{Specimen-specific finite element models}

To determine the material constants of each osteochondral plug, specimen-specific FEMs were created in a commercial finite-element software package (Abaqus v 6.11, Simulia Corp., Providence, RI) (see Fig. 2f). The reconstructed geometry of each specimen was imported and meshed with hybrid quadratic tetrahedral elements (Abaqus element type C3D10H) using an element size of approximately $0.25 \mathrm{~mm}$. The face of the bone glued to the stainless steel rod was represented as a clamped boundary condition and frictionless contact was modeled between the articular cartilage surface and the glass plate. The glass plate was modeled as a rigid surface.

The FEM was run quasi-statically with large deformation under displacement control. For the simulations considering a neo-Hookean cartilage model, the values of $\mu$ and $\mathrm{E}_{\mathrm{SB}+\mathrm{CC}}$ were 
determined by solving an iterative optimization problem that minimized the following least squares function:

$f=\sum_{i=1}^{n}\left[\left(\frac{F_{\exp , i}-F_{\text {sim }, i}}{\max \left(F_{\exp }\right)}\right)^{2}+\left(\frac{\varepsilon_{\exp , i}-\varepsilon_{\text {sim }, i}}{\max \left(\varepsilon_{\exp }\right)}\right)^{2}\right]$

where $i$ represents the time increment; $\mathrm{n}$ is the final time increment; $\mathrm{F}_{\text {exp, }}$ is the experimentally measured contact force; $\mathrm{F}_{\text {sim,i i }}$ is the contact force predicted by the FEM; $\varepsilon_{\text {exp,i }}$ is the maximum axial strain along the edges of the $\mathrm{SB}+\mathrm{CC}$ (Eqs. 1 and 2 above); and $\varepsilon_{\text {sim,i }}$ is the corresponding axial engineering strain predicted by the FEM. When implementing the Yeoh cartilage model the value of $\mathrm{E}_{\mathrm{SB}+\mathrm{CC}}$ was equated to that calculated in the aforementioned optimization problem, whereas the values of $\mathrm{C}_{10}$ and $\mathrm{C}_{20}$ were found by solving another optimization problem that minimized the least squares function:

$f=\sum_{i=1}^{n}\left(\frac{F_{\text {exp }, i}-F_{\text {sim }, i}}{\max \left(F_{\text {exp }}\right)}\right)^{2}$

In these optimization problems, the material parameters $C_{30}, K_{0}, D_{1}, D_{2}$ and $D_{3}$ were either set to zero or neglected due to the assumption of incompressibility. Initial guesses for $\mu, \mathrm{C}_{10}$ and $\mathrm{C}_{20}$ were based on literature data (Anderson et al., 2008; Brown et al., 2009). Model sensitivity to the initial values of these variables and the convergence of the finite element mesh were each evaluated by calculating $\mu, \mathrm{C}_{10}$ and $\mathrm{C}_{20}$ for a representative specimen over a range of initial values, mesh densities and element types. The calculated values of $\mu, \mathrm{C}_{10}$ and $\mathrm{C}_{20}$ each changed by less than $1 \%$, indicating that the optimization solution was insensitive to the initial values assumed for these variables and that there was good mesh convergence. 


\subsection{Statistical Analyses}

Specimens with an OARSI grading of 0 to 1 (i.e., specimens showing no damage or only mild damage) were treated as controls while those with an OARSI grade of 2 and above (i.e., specimens exemplifying signs of macrodamage such as fibrillation, erosion and cracking) constituted the OA group. The mean and standard deviation (SD) for experimental and computational parameters were calculated for the control specimens and OA specimens by fitting a linear-mixed model (Brown and Prescott, 2006). This model accounts for random effects that may arise from subject-specific dependencies or from tissue acquired from different sources (i.e., cadaveric specimens versus in vivo specimens acquired prior to TKA). The linear-mixed model was also used to test for significant differences of measured variables between the control and OA groups. For each variable, scatter plots of the residuals versus the fitted values and a histogram of the residuals were used to confirm, respectively, linearity and normality assumed by the linear-mixed model. For the correlation analyses, all 84 specimens were pooled and correlations with structural parameters ( $\mathrm{t}_{\text {cart, }}$ zonal thicknesses and PG thickness) and OARSI grade were assessed using Pearson's correlation coefficients. Contact force, average contact pressure, and contact area obtained from the specimen-specific FEM simulations were evaluated against the corresponding experimental results by computing the respective RMS errors. Significant differences in RMS errors between the neo-Hookean and Yeoh models were assessed using a two-sample Kolmogorov-Smirnov test.

\section{Results}

The shear modulus of the pooled control specimens $(6.0 \pm 1.6 \mathrm{MPa})$ was significantly higher $(p<0.05)$ than that of the pooled OA specimens $(4.6 \pm 1.8 \mathrm{MPa})$ (Table 1 ; see also supplementary 
material Table 1). The shear modulus of the tibial control specimens was significantly higher $(\mathrm{p}<0.05)$ than that of the tibial OA specimens. In contrast, the shear modulus of the femoral control specimens was not significantly different from that of the femoral OA specimens. The Yeoh material constants, $\mathrm{C}_{10}$ and $\mathrm{C}_{20}$, for the pooled controls $(1.7 \pm 0.8 \mathrm{MPa}$ and $3.9 \pm 3.4 \mathrm{MPa}$, respectively) were significantly higher $(\mathrm{p}<0.05$, respectively) than those for the pooled OA specimens (1.1 $\pm 0.8 \mathrm{MPa}$ and 2.0 $\pm 1.5 \mathrm{MPa}$, respectively). The PG thickness was significantly greater for the pooled control specimens than the pooled OA specimens $(91.0 \pm 5.7 \%$ and $86.2 \pm 8.7 \%$, respectively) $(\mathrm{p}<0.05)$. The values measured for cartilage thickness, zonal thicknesses, and $\mathrm{E}_{\mathrm{SB}+\mathrm{CC}}$ were not significantly different between the pooled control and pooled OA specimens (Table 1). The Cohen's kappa coefficient was 0.31 , indicating a fair interobserver agreement in the OARSI grades.

Significant correlations were observed between the hyperelastic material constants and the structural parameters and OARSI grade of the cartilage (Table 2 and Fig. 7). $\mu, \mathrm{C}_{10}$ and $\mathrm{C}_{20}$ each correlated with OARSI grade $(\mathrm{R}=-0.55, \mathrm{p}<0.001 ; \mathrm{R}=-0.45, \mathrm{p}<0.001$; and $\mathrm{R}=-0.35, \mathrm{p}<0.001$; respectively). $\mu$ and $\mathrm{C}_{10}$ each correlated significantly with cartilage thickness as well as the intermediate and deep zone thicknesses.

For both the pooled control and pooled OA specimens the neo-Hookean model over-predicted the mean contact force at low cartilage strains and under-predicted this quantity at high cartilage strains (Fig. 8). By comparison, the Yeoh model accurately reproduced the mean contact force at all cartilage strains. These trends were also evident for the average contact pressure. Both models under-predicted the mean contact area at all cartilage strains. 
The radial strain ratios determined experimentally for the pooled control and pooled OA specimens were greater than 1.0, indicating some degree of anisotropy within the superficial zone for all specimens tested (Fig. 8). When pooled from all increments of axial cartilage strain, the radial strain ratio for the pooled control specimens (2.6 \pm 1.2$)$ was significantly higher $(\mathrm{p}<0.05)$ than that for the pooled OA specimens $(2.1 \pm 1.0)$. Although the radial strain ratios predicted by both the neo-Hookean and Yeoh models were slightly above 1.0, each model underpredicted the values determined from experiment across all axial strains of the cartilage (Fig. 8). The maximum axial strain on the edges of the $\mathrm{SB}+\mathrm{CC}$ was less than $13 \%$ for the pooled control specimens and less than $16 \%$ for the pooled OA specimens (Fig. 8). The linear-elastic model described the axial strains of the $\mathrm{SB}+\mathrm{CC}$ accurately for the pooled OA specimens, and slightly over-predicted these strains for the pooled control specimens.

RMS errors in contact force and average contact pressure were significantly lower for the Yeoh model than the neo-Hookean model for both the pooled control $(\mathrm{p}<0.001)$ and pooled OA specimens $(\mathrm{p}<0.001)$ (Fig. 9). RMS errors for contact area were not significantly different between the Yeoh and neo-Hookean models for both the pooled control and pooled OA specimens.

\section{Discussion}

We measured the hyperelastic material constants of normal and osteoarthritic knee cartilage using two common constitutive models, the neo-Hookean and Yeoh models, and quantified the ability of each model to describe measurements of contact force, contact area, average contact pressure, and anisotropy of the tissue constituting the superficial zone. We also examined whether the hyperelastic material constants correlated with the structural parameters (e.g., zonal 
thicknesses) of the tissue and the severity of cartilage damage as determined from the OARSI grading scale. Isotropic hyperelastic models have previously been used to study the mechanical behavior of cartilage at the human hip (Anderson et al., 2008, Henak et al., 2014), human elbow (Willing et al., 2013), human jaw (Koolstra and Van Eijden 2006), porcine knee (Butz et al., 2011), and bovine patella (Brown et al., 2009). The current study presents novel data for the material constants of osteoarthritic human knee cartilage derived from isotropic hyperelastic models.

The hyperelastic constants of the neo-Hookean model $(\mu)$ and Yeoh model $\left(\mathrm{C}_{10}\right.$ and $\left.\mathrm{C}_{20}\right)$ were significantly higher in the control specimens, implying higher cartilage stiffness (Table 1). The lower values of the hyperelastic material constants for the OA specimens are most likely a consequence of increased cartilage damage associated with advanced OA (OARSI grade of 2 and above). The shear modulus of the tibial cartilage was higher for the control specimens compared to the OA specimens, but this trend was not observed for the femoral cartilage (Table 1). This finding may be related to differences in PG content, since the PG thickness also varied significantly between the tibial control and tibial OA specimens, and the pooled control and pooled OA specimens. The difference in shear modulus between the tibial and femoral cartilage may also suggest that the mechanical properties of tibial cartilage experience greater change with increasing tissue damage compared to femoral cartilage, but caution is advised in interpreting this result as the number of tibial control specimens was relatively low $(n=10)$. Interestingly, ESB+CC Was not significantly different between the pooled control and pooled OA specimens. This finding may be a consequence of calculating the combined Young's modulus for the subchondral bone and calcified cartilage layers. Hence, it was not possible to determine whether the respective modulus of these layers changed with increasing OA, as reported in other studies 
(Day et al., 2001; Ferguson et al., 2003; Hargrave-Thomas et al., 2015). The hyperelastic constants determined in the present study may be used in future investigations that apply isotropic and homogeneous models to simulate the mechanical behavior of normal and osteoarthritic cartilage at the tibiofemoral joint.

The present study is the first to investigate whether the hyperelastic material constants of normal and osteoarthritic cartilage correlate with structural parameters and/or tissue damage. Using a linear regression, a number of significant correlations were observed with Pearson's correlation coefficients ranging between -0.55 to 0.34 (Table 2 ). The hyperelastic material constants correlated most strongly with OARSI grade, a histopathological measure of tissue damage. These correlations establish that the mechanical properties of cartilage determined under large compressive strains and 'instantaneous' loading rates are significantly altered by tissue damage.

Although the contact areas of cartilage explants undergoing unconfined compression have been measured previously (Demarteau et al., 2006), images of the contact area at 'instantaneous' loading rates representative of physiological activity have not been reported. Using these images we confirmed that the superficial zone of cartilage is anisotropic. This is an important finding because anisotropy is often neglected in models of whole joints undergoing 'instantaneous' loading (Anderson et al., 2008; Haut Donahue et al., 2003; Henak et al., 2014; Papaioannou et al., 2008). The degree of anisotropy of cartilage in the superficial zone varied considerably between specimens and was significantly higher in the control specimens compared to the OA specimens (Fig. 8). Since anisotropy of cartilage is primarily due to the arrangement of the collagen fibril network (Bachrach et al., 1998), disruption of the network due to tissue damage may explain the lower degree of anisotropy observed in the OA specimens. Changes in cartilage anisotropy may affect the mechanical behavior of the superficial zone chondrocytes in view of 
the finding that these chondrocytes exhibit direction-dependent properties relative to the local split-line directions (Guilak et al., 1995).

Comparing the abilities of the two hyperelastic models to accurately reproduce measurements of the contact variables, the Yeoh model described the magnitude and shape of the contact force and average contact pressure more accurately than the neo-Hookean model. This is consistent with the knowledge that the latter model is characterized by a second-order strain energy function with a larger number of material constants. Nonetheless, the neo-Hookean model predicted contact force to an accuracy of $7 \%$ and its material constant correlated more strongly with OARSI grade (Table 2), suggesting that this model may be better suited to representing the mechanical behavior of normal and osteoarthritic cartilage. Mean RMS errors in the average contact pressures predicted by the neo-Hookean and Yeoh models ranged from $10 \%$ to $19 \%$ and each model under-predicted contact area with mean RMS errors of $17 \%$ to $20 \%$ (Fig. 9). As expected, both models poorly described the anisotropy of cartilage within the superficial zone (Fig. 8). These results highlight the limits to which simple isotropic, hyperelastic, material models may be used to describe measurements of contact force, average contact pressure, contact area and anisotropy. If greater accuracy in model predictions is needed in future work, then anisotropic models and/or an inhomogeneous material with a reduced stiffness at the surface may yield better descriptions of cartilage contact variables.

\subsection{Comparison with previous studies}

The values of the shear modulus measured for the controls (femur: $5.7 \pm 1.8 \mathrm{MPa}$; tibia: $6.2 \pm 1.1$ $\mathrm{MPa}$ ) are similar to results reported for healthy hip cartilage using the neo-Hookean model (femoral head: 4.9 $\pm 2.0 \mathrm{MPa}$; acetabulum: 6.4 $\pm 2.6 \mathrm{MPa}$ ) (Henak et al., 2014). Whilst it is not ideal to compare cartilage properties between different joints, previous studies have also noted 
similarities between the 'instantaneous' compressive moduli for knee and hip cartilage (Shepherd and Seedhom, 1999). It is not possible to compare the hyperelastic constants of the pooled OA specimens to other studies as these quantities have not been reported for human osteoarthritic cartilage.

Our estimate of $\mathrm{E}_{\mathrm{SB}+\mathrm{CC}}$ for the pooled control specimens (mean value of $446.6 \mathrm{MPa}$; Table 1) is within the range of values reported by Mente and Lewis (1994) for the Young's moduli of the individual layers of subchondral bone and calcified cartilage (5.7 GPa and $320 \mathrm{MPa}$, respectively). Unfortunately, it is difficult to compare our estimate of $\mathrm{E}_{\mathrm{SB}+\mathrm{CC}}$ for the pooled $\mathrm{OA}$ specimens against these results because Mente and Lewis (1994) did not evaluate the condition of the joints used in their study.

The mean radial strain ratios measured for the pooled control and pooled OA specimens were greater than 2.0 (Fig. 8), which is consistent with results reported for elderly human and bovine cartilage (mean values of 3.1 and 2.2, respectively) (Demarteau et al., 2006). Differences between these results and those obtained in the present study may have resulted from dissimilar loading protocols. Demarteau et al., (2006) applied a series of 5\% axial compressive strain increments with a 30-minute stress relaxation period used between each loading increment, whereas the current study used one increment of $30 \%$ axial strain with no stress relaxation.

The correlations between the hyperelastic constants and OARSI grade are consistent with the results of Armstrong and Mow (1982), Kiviranta et al., (2008), and Kleemann et al., (2005) who found that both the equilibrium and dynamic Young's modulus decreased with histopathological cartilage damage. Significant correlations were also observed between the hyperelastic constants and cartilage thickness, which has not been previously reported. It is well documented that in 
early OA, cartilage experiences hypertrophy due to a change in its swelling characteristics caused by PG depletion (Calvo et al., 2004; Pritzker et al., 2006). Hypertrophy increases the thickness of cartilage whereas PG depletion softens the tissue (Korhonen et al., 2003). This phenomenon may explain the negative correlation with cartilage thickness for the specimens with less severe damage (OARSI grades 0-1), but it does not account for this trend in the more severely damaged specimens. The negative correlation may be due in part to inexact modeling of the experimental boundary condition. In the current experiments, the clamped boundary condition may have been less rigid in those specimens having a greater height-to-diameter ratio, perhaps due to bending, and this effect may not have been adequately modeled in the FEMs. Further experimental evidence is needed to verify this reasoning.

The relatively weak correlations between cartilage mechanical properties and the PG thickness and zonal thicknesses contradict previous findings that the equilibrium or dynamic Young's modulus is strongly correlated to PG and collagen properties (Kiviranta et al., 2008, 2006). In the current study, zonal thicknesses and the relative PG thickness were each measured in histological sections. The PG thickness was assumed to be representative of changes in PG content as previous work has shown that PG content progressively decreases with depth below the articular surface as OA severity increases (Saarakkala et al., 2010). In contrast, the correlations reported by Kiviranta et al., (2008, 2006) were based upon PG and collagen content as well as average specimen birefringence as determined by quantitative microscopic analysis techniques. The use of these different structural parameters may have contributed to stronger correlations than those obtained in the present study.

The trends in contact force obtained from the constitutive models are consistent with those reported by Henak et al., (2014). These authors found that the neo-Hookean Cauchy stress 
(which was calculated from contact force) was over-predicted at low compressive strains and under-predicted at high strains, and they also reported that a hyperelastic constitutive model with two independent constants, the Veronda Westmann model, described Cauchy stress more accurately.

\subsection{Limitations of the analysis}

The present study is not without limitations. First, the pooled control and pooled OA groups comprised of specimens from two different sources, cadaveric knees and tissue harvested from patients undergoing TKA surgery. However, our statistical analysis accounted for these differences by representing them as random effects in a linear-mixed model. Furthermore, covariance estimates obtained using the linear-mixed model indicated that for all experimental and computational parameters, the variance due to within-subject effects was larger than that due to either between-subject effects or the use of cartilage harvested from two different sources. The finding that within-subject effects had a large influence on the measured parameters justifies combining the cadaveric and TKA specimens for the statistical analyses.

Second, the boundary conditions associated with the unconfined compression experiments are presumably different from those encountered by cartilage in vivo. In the absence of a reasonable alternative, unconfined compression remains the most common method by which anisotropic or inhomogenous cartilage properties are obtained across a range of loading rates (Demarteau et al., 2006; Jurvelin et al., 1997; Kiviranta et al., 2006; Wang et al., 2003, Wong et al., 2000). It should be noted, however, that the layer of $\mathrm{SB}+\mathrm{CC}$ was retained, thus representing the physiological conditions at this tidemark interface. We also added a thin film of PBS at the articular surface to reduce friction during the loading experiments, but the effectiveness of this film cannot be fully quantified. To further investigate the effects of friction in our experiments, 
friction was added to the FEM of one specimen with frictional coefficients ranging from 0 (frictionless condition) to a value of 0.1 (moderate). Over this range the shear modulus decreased by $3.6 \%$, from $4.54 \mathrm{MPa}$ to $4.38 \mathrm{MPa}$, supporting our contention that the experimental results are relatively insensitive to friction.

Third, although our 3D geometry acquisition method was shown to be accurate for the rubber and epoxy laminate, the accuracy with which it was able to reproduce the osteochondral plug geometry is unknown. The contrast between the cartilage tissue and the background was similar to that seen for the rubber specimens, and the acquisition of its outer surface was likely accurate. However, at some viewing angles the tidemark was not visible and may have led to the inclusion of some portion of the calcified cartilage layer in the cartilage geometry. A sensitivity analysis was performed on one specimen where, as a worst-case scenario, the entire calcified cartilage layer of an estimated thickness of $0.17 \mathrm{~mm}$ (Mente and Lewis 1994) was added to the cartilage geometry. The shear modulus was found to increase by $7.5 \%$, from $4.54 \mathrm{MPa}$ to $4.88 \mathrm{MPa}$, suggesting that the FEM methods used to evaluate the material constants are moderately sensitive to errors in the tidemark position. This change in shear modulus represents the maximum error. Because the tidemark was distinguishable at most viewing angles, smaller errors are likely to be associated with the position of the tidemark, and hence the calculated values of the material constants.

Fourth, the present study did not investigate whether other variables such as stress and strain are associated with different amounts of tissue damage. There is evidence to suggest that collagen damage may result from excessive collagen fibril strains or shear applied in the direction of the fibrils (Wilson et al., 2006), and that intermittent hydrostatic compressive stress and octahedral shear stress inhibits ossification of cartilage tissue (review by Carter et al., 2004). Our 
measurements of radial strains at the articular surface and total axial strain do not yield reliable estimates of depth-dependent cartilage strains, and so relationships between stress/strain and damage could not be quantified.

Fifth, the isotropic, homogeneous, hyperelastic material models used in the present study are limited in their ability to describe intrinsic cartilage properties, such as anisotropy, inhomogeneity and tension-compression nonlinearity (see review by Mow and Guo 2002), hence the material constants given in Table 1 represent apparent material properties. However, since the FEMs accurately incorporated specimen-specific geometry and boundary conditions, the resulting material constants were decoupled from our testing methods and may be used in future applications to describe cartilage contact mechanics where the tissue is assumed to be isotropic and homogeneous. Due to the instantaneous loading rates, cartilage was considered an incompressible elastic material and effects such as fluid flow were neglected, as assumed in other studies (Henak et al., 2015; Pierce et al., 2009). However, this does limit the applicability of our results to intervals of high loading rates, for example, during the stance phases of walking and running.

Sixth, due to the high number of subject-specific FEMs, our analysis was restricted to two hyperelastic models. The neo-Hookean model was chosen because it is characterized by a simple hyperelastic strain energy function, which is one reason why it has been previously used to describe articular cartilage (Anderson et al., 2008; Henak et al., 2014). The Yeoh model was chosen because its strain energy function has a nonlinear dependence on strain, consistent with another isotropic hyperelastic model, the Veronda Westmann model, which was used by Henak et al. (2014) to describe cartilage material nonlinearities. In addition, the Yeoh model is widely available in commercial finite-element software packages such as Abaqus. 
In summary, the results of this study provide novel data for the material constants of isotropic hyperelastic models of normal and osteoarthritic human knee cartilage. The data show that hyperelastic material constants are altered in a progressive nature with damage to the cartilage.

These results have applications in whole-joint models that describe cartilage with varying degrees of tissue damage. Differences between two commonly used hyperelastic material models, the neo-Hookean and Yeoh models, were quantified, and the results suggest the limits to which these simple isotropic hyperelastic models may be used to study cartilage contact mechanics.

\section{Acknowledgements}

This work was supported by a Discovery Projects grant from the Australian Research Council (DP120101973) and an Innovation Fellowship from the Victorian Endowment for Science, Knowledge and Innovation to MGP. St Vincent's Institute of Medical research is supported in part by the Victorian State Government Operational Infrastructure Support Programme.

\section{References}

Anderson, A.E., Ellis, B.J., Maas, S.A., Peters, C.L. and Weiss, J.A. (2008). Validation of finite element predictions of cartilage contact pressure in the human hip joint. J Biomech Eng 130(5):051008.

Armstrong, C.G. and Mow, V.C. (1982). Variations in the intrinsic mechanical properties of human articular cartilage with age, degeneration, and water content. J Bone Joint Surg Am 64(1):88-94.

Ashman, R.B., Cowin, S.C., Van Buskirk, W.C. and Rice, J.C. (1984). A continuous wave technique for the measurement of the elastic properties of cortical bone. J Biomech 17(5):349-361.

Ateshian, G.A., Ellis, B.J. and Weiss, J.A. (2007). Equivalence between short-time biphasic and incompressible elastic material responses. J Biomech Eng, 129(3):405-412.

Ateshian, G.A. and Mow, V.C. (2005). Friction, lubrication, and wear of articular cartilage and diarthrodial joints. In Mow, V.C. and Huiskes, R., editors, Basic orthopaedic 
biomechanics and mechano-biology, 3rd edition 447-494. Lippincott Williams \& Wilkins.

Athanasiou, K., Agarwal, A. and Dzida, F. (1994). Comparative study of the intrinsic mechanical properties of the human acetabular and femoral head cartilage. J Orthop Res 12(3):340349.

Bachrach, N.M., Mow, V.C. and Guilak, F. (1998). Incompressibility of the solid matrix of articular cartilage under high hydrostatic pressures. J Biomech 31(5):445-451.

Bi, X., Li, G., Doty, S.B. and Camacho, N.P. (2005). A novel method for determination of collagen orientation in cartilage by Fourier transform infrared imaging spectroscopy (FTIRIS). Osteoarthritis Cartilage 13(12):1050-1058.

Bingham, J.T., Papannagari, R., Van de Velde, S.K., Gross, C., Gill, T.J., Felson, D.T., Rubash, H.E. and Li, G. (2008). In vivo cartilage contact deformation in the healthy human tibiofemoral joint. Rheumatology 47(11):1622-1627.

Brown, C.P., Nguyen, T.C., Moody, H.R., Crawford, R.W. and Oloyede, A. (2009). Assessment of common hyperelastic constitutive equations for describing normal and osteoarthritic articular cartilage. Proc Inst Mech Eng H 223(6):643-652.

Brown, H. and Prescott, R. (2006). Normal mixed models. In Applied mixed models in medicine, 2nd edition 33-105. John Wiley \& Sons.

Butz, K.D., Chan, D.D., Nauman, E.A. and Neu, C.P. (2011). Stress distributions and material properties determined in articular cartilage from MRI-based finite strains. J Biomech 44(15):2667-2672.

Calvo, E., Palacios, I., Delgado, E., Sanchez-Pernaute, O., Largo, R., Egido, J. and HerreroBeaumont, G. (2004). Histopathological correlation of cartilage swelling detected by magnetic resonance imaging in early experimental osteoarthritis. Osteoarthritis Cartilage 12(11):878-886.

Carter, D.R., Beaupré, G.S., Wong, M., Smith R.L., Andriacchi T.P. and Schurman D.J. (2004). The mechanobiology of articular cartilage development and degeneration. Clin Orthop Relat Res, 427:S69-S77.

Changoor, A., Fereydoonzad, L., Yaroshinsky, A. and Buschmann, M.D. (2010). Effects of refrigeration and freezing on the electromechanical and biomechanical properties of articular cartilage. J Biomech Eng 132(6):064502.

Day, J.S., Ding, M., Van der Linden, J.C., Hvid, I., Sumner, D.R. and Weinans, H. (2001). A decreased subchondral trabecular bone tissue elastic modulus is associated with pre-arthritic cartilage damage. J Orthop Res 19(5):914-918.

Demarteau, O., Pillet, L., Inaebnit, A., Borens, O. and Quinn, T.M. (2006). Biomechanical characterization and in vitro mechanical injury of elderly human femoral head cartilage: comparison to adult bovine humeral head cartilage. Osteoarthritis Cartilage 14(6):589596.

Ferguson, V.L., Bushby, A.J. and Boyde A. (2003). Nanomechanical properties and mineral concentration in articular calcified cartilage and subchondral bone. J Anat, 203(2):191202.

Guilak, F., Ratcliffe, A. and Mow, V.C. (1995). Chondrocyte deformation and local tissue strain in articular cartilage: A confocal microscopy study. J Orthop Res, 13(3):410-421.

Haut, T.L., Hull, M.L. and Howell, S.M. (1998). A high-accuracy three-dimensional coordinate digitizing system for reconstructing the geometry of diarthrodial joints. J Biomech 31(6):571-577. 
Haut Donahue, T.L., Hull, M., Rashid, M.M. and Jacobs, C.R. (2003). How the stiffness of meniscal attachments and meniscal material properties affect tibio-femoral contact pressure computed using a validated finite element model of the human knee joint. $\mathbf{J}$ Biomech 36(1):19-34.

Hargrave-Thomas, E., van Sloun, F., Dickinson, M., Broom, N. and Thambyah, A. (2015). Multi-scalar Mechanical Testing of the Calcified Cartilage and Subchondral Bone Comparing Healthy versus Early Degenerative states. Osteoarthritis Cartilage 23(10):1755-1762.

Henak, C.R., Kapron, A.L., Anderson, A.E., Ellis, B.J., Maas, S.A. and Weiss, J.A. (2014). Specimen-specific predictions of contact stress under physiological loading in the human hip: validation and sensitivity studies. Biomech Model Mechanobiol 13(2):387-400.

Jurvelin, J.S., Buschmann, M.D. and Hunziker, E.B. (1997). Optical and mechanical determination of Poisson's ratio of adult bovine humeral articular cartilage. J Biomech, 30(3):235-241.

Kiapour, A., Kiapour, A.M., Kaul, V., Quatman, C.E., Wordeman, S.C., Hewett, T.E., Demetropoulos, C.K. and Goel, V.K. (2014). Finite element model of the knee for investigation of injury mechanisms: development and validation. J Biomech Eng 136(1):011002.

Kiefer, G.N., Sundby, K., McAllister, D., Shrive, N.G., Frank, C.B., Lam, T. and Schachar, N.S. (1989). The effect of cryopreservation on the biomechanical behavior of bovine articular cartilage. Journal Orthop Res 7(4):494-501.

Kiviranta, P., Lammentausta, E., Töyräs, J., Kiviranta, I. and Jurvelin, J.S. (2008). Indentation diagnostics of cartilage degeneration. Osteoarthritis Cartilage 16(7):796-804.

Kiviranta, P., Rieppo, J., Korhonen, R.K., Julkunen, P., Töyräs, J. and Jurvelin, J.S. (2006). Collagen network primarily controls Poisson's ratio of bovine articular cartilage in compression. J Orthop Res 24(4):690-699.

Kleemann, R.U., Krocker, D., Cedraro, A., Tuischer, J. and Duda, G.N. (2005). Altered cartilage mechanics and histology in knee osteoarthritis: relation to clinical assessment (ICRS Grade). Osteoarthritis Cartilage 13(11):958-963.

Koolstra, J.H. and Van Eijden, T.M.G.J. (2006). Prediction of volumetric strain in the human temporomandibular joint cartilage during jaw movement. J Anat, 209(3):369-380.

Korhonen, R.K., Laasanen, M.S., Töyräs, J., Lappalainen, R., Helminen, H.J. and Jurvelin, J.S. (2003). Fibril reinforced poroelastic model predicts specifically mechanical behavior of normal, proteoglycan depleted and collagen degraded articular cartilage. J Biomech 36(9):1373-1379.

Liu, F., Kozanek, M., Hosseini, A., Van de Velde, S.K., Gill, T.J., Rubash, H.E. and Li, G. (2010). In vivo tibiofemoral cartilage deformation during the stance phase of gait. J Biomech 43(4):658-665.

Mente, P.L. and Lewis, J.L. (1994). Elastic modulus of calcified cartilage is an order of magnitude less than that of subchondral bone. J Orthop Res 12(5):637-647.

Morel, V. and Quinn, T.M. (2004). Cartilage injury by ramp compression near the gel diffusion rate. J Orthop Res 22(1):145-151.

Mow, V.C. and Guo, X.E. (2002). Mechano-electrochemical properties of articular cartilage: their inhomogeneities and anisotropies Annu Rev Biomed Eng 4:175-209. 
Oloyede, A., Flachsmann, R. and Broom, N.D. (1992). The dramatic influence of loading velocity on the compressive response of articular cartilage. Connect Tissue Res 27(4):211-224.

Papaioannou, G., Nianios, G., Mitrogiannis, C., Fyhrie, D., Tashman, S. and Yang, K.H. (2008). Patient-specific knee joint nite element model validation with high-accuracy kinematics from biplane dynamic Roentgen stereogrammetric analysis. J Biomech, 41(12):26332638.

Pierce, D.M., Trobin, W., Trattnig, S., Bischof, H. and Holzapfel,G.A. (2009). A phenomenological approach toward patient-specific computational modeling of articular cartilage including collagen fiber tracking. J Biomech Eng, 131(9):091006.

Pritzker, K.P., Gay, S., Jimenez, S.A., Ostergaard, K., Pelletier, J.P., Revell, P.A., Salter, D. and van den Berg, W.B. (2006). Osteoarthritis cartilage histopathology: grading and staging. Osteoarthritis Cartilage 14(1):13-29.

Rieppo, J., Töyräs, J., Nieminen, M.T., Kovanen, V., Hyttinen, M.M., Korhonen, R.K., Jurvelin, J.S. and Helminen, H.J. (2003). Structure-function relationships in enzymatically modified articular cartilage. Cells Tissues Organs 175(3):121-132.

Rosenberg, L., (1971). Chemical basis for the histological use of safranin O in the study of articular cartilage. J Bone Joint Surg Am 53(1):69-82.

Saarakkala, S., Julkunen, P., Kiviranta, P., Mäkitalo, J., Jurvelin, J.S. and Korhonen, R.K. (2010). Depth-wise progression of osteoarthritis in human articular cartilage: investigation of composition, structure and biomechanics. Osteoarthritis Cartilage 18(1):73-81.

Saarakkala, S., Laasanen, M.S., Jurvelin, J.S., Törrönen, K., Lammi, M.J., Lappalainen, R. and Töyräs, J. (2003). Ultrasound indentation of normal and spontaneously degenerated bovine articular cartilage. Osteoarthritis Cartilage 11(9):697-705.

Salai, M., Givon, U., Messer, Y. and von Versen, R. (1997). Electron microscopic study on the effects of different preservation methods for meniscal cartilage. Ann Transplant 2(1):5254.

Setton, L.A., Elliott, D.M. and Mow, V.C. (1999). Altered mechanics of cartilage with osteoarthritis: human osteoarthritis and an experimental model of joint degeneration. Osteoarthritis Cartilage 7(1):2-14.

Shepherd, D.E. and Seedhom, B.B. (1999). The 'instantaneous' compressive modulus of human articular cartilage in joints of the lower limb. Rheumatology 38(2):124-132.

Szarko, M., Muldrew, K. and Bertram, J.E. (2010). Freeze-thaw treatment effects on the dynamic mechanical properties of articular cartilage. BMC Musculoskelet Disord 11(1):231.

Wang, C.C., Chahine, N.O., Hung, C.T. and Ateshian, G.A. (2003). Optical determination of anisotropic material properties of bovine articular cartilage in compression. J Biomech, 36(3):339-353.

Wong, M., Ponticiello, M., Kovanen, V. and Jurvelin, J.S. (2000). Volumetric changes of articular cartilage during stress relaxation in unconfined compression. J Biomech, 33(9):1049-1054.

Willing, R.T., Lalone, E.A., Shannon, H., Johnson, J.A. and King, G.J. (2013). Validation of a finite element model of the human elbow for determining cartilage contact mechanics. $\mathbf{J}$ Biomech 46(10):1767-1771. 
Wilson, W., van Burken, C., van Donkelaar, C., Buma, P., van Rietbergen, B. and Huiskes, R. (2006). Causes of mechanically induced collagen damage in articular cartilage. J Orthop Res 24(2):220-228. 
Table 1 Mean \pm 1 SD of experimental and computational parameters describing the mechanical and structural properties of the control and OA cartilage. All data were obtained from $3 \mathrm{~mm}$ diameter osteochondral plugs extracted from the tibial plateau and femoral condyles of human knees. Significant differences between groups are indicated with $\mathrm{P}<0.05$.

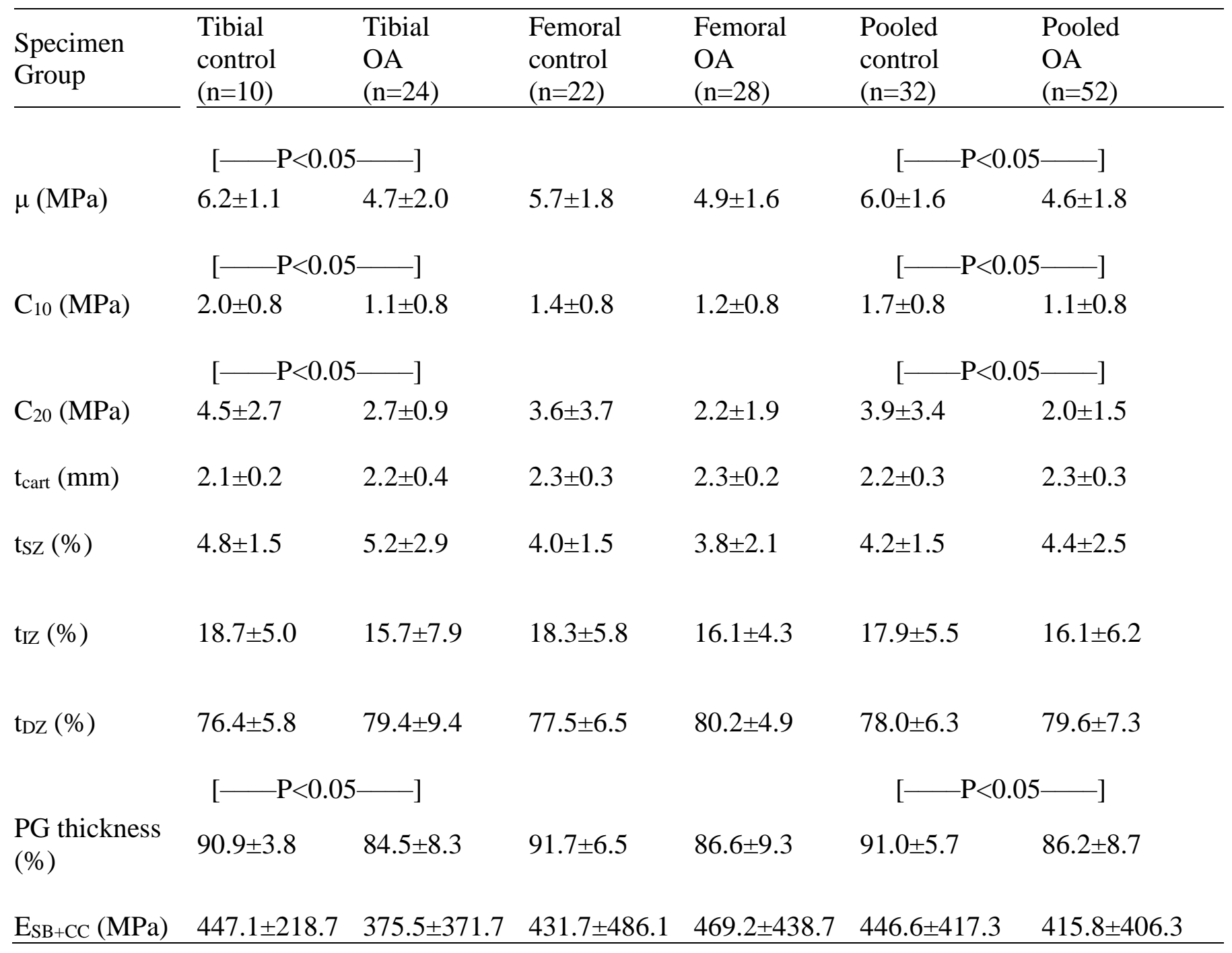

$\mu$, infinitesimal shear modulus; $\mathrm{C}_{10}$ and $\mathrm{C}_{20}$, Yeoh model material constants; $\mathrm{t}_{\text {cart }}$, cartilage thickness; $t_{\mathrm{SZ}}, \mathrm{t}_{\mathrm{IZ}}$, and $\mathrm{t}_{\mathrm{DZ}}$, thicknesses of the superficial, intermediate and deep zones, respectively, each normalized by $t_{\text {cart }}$; PG thickness, thickness of proteoglycan normalized by $\mathrm{t}_{\text {cart }}$; $\mathrm{E}_{\mathrm{SB}+\mathrm{CC}}$, Young's modulus of the combined layer of subchondral bone and calcified cartilage. 
Table 2 Pearson's correlation coefficients describing relationships between the mechanical properties of cartilage $\left(\mu, \mathrm{C}_{10}\right.$, and $\left.\mathrm{C}_{20}\right)$ and cartilage structural parameters $\left(\mathrm{t}_{\mathrm{car}}, \mathrm{t}_{\mathrm{sZ}}, \mathrm{t}_{\mathrm{zZ}}, \mathrm{t}_{\mathrm{DZ}} \mathrm{PG}\right.$ thickness) and OARSI grade. All data were calculated with the control and OA specimens pooled together.

\begin{tabular}{llll}
\hline Parameter & \multicolumn{3}{l}{ Pooled specimens $(\mathrm{n}=84)$} \\
\cline { 3 - 4 } & $\mu$ & $\mathrm{C}_{10}$ & $\mathrm{C}_{20}$ \\
\cline { 3 - 4 } $\mathrm{t}_{\text {cart }}$ & $-0.38^{* *}$ & $-0.29 *$ & -0.13 \\
$\mathrm{t}_{\mathrm{SZ}}$ & 0.15 & 0.09 & -0.02 \\
$\mathrm{t}_{\mathrm{IZ}}$ & $0.34^{*}$ & $0.26^{*}$ & -0.01 \\
$\mathrm{t}_{\mathrm{DZ}}$ & $-0.34^{*}$ & $-0.25^{*}$ & 0.01 \\
PG thickness & -0.01 & 0.04 & 0.08 \\
OARSI grade & $-0.55^{* *}$ & $-0.45^{* *}$ & $-0.35^{* *}$ \\
\hline
\end{tabular}

$\mu$, infinitesimal shear modulus; $\mathrm{C}_{10}$ and $\mathrm{C}_{20}$, Yeoh model material constants; $\mathrm{t}_{\text {cart, }}$ cartilage thickness; $\mathrm{tsz}_{\mathrm{s}} \mathrm{t}_{\mathrm{z}}$, and $\mathrm{t}_{\mathrm{DZ}}$, thicknesses of the superficial, intermediate and deep zones, respectively, each normalized by $t_{\text {cart }}$; PG thickness, proteoglycan thickness normalized by $t_{c a r t}$; OARSI grade, degree of cartilage damage.

$* \mathrm{P}<0.05$.

$* * \mathrm{P}<0.001$. 


\section{Figure Captions}

Fig. 1 Schematic diagram illustrating the method used for specimen preparation. a A $5 \mathrm{~mm}$ diameter cylindrical osteochondral specimen was extracted with a coring device held normal to the articular surface of the femoral condyle or tibial plateau. b An inner $3 \mathrm{~mm}$ core was extracted with a dermal punch and $5 \mu \mathrm{m}$ sections were cut from one side of the specimen for histological analysis. Grey shading Articular cartilage. Brown shading Subchondral bone and calcified cartilage. c Geometry of a $3 \mathrm{~mm}$ osteochondral plug used for mechanical testing. d $5 \mu \mathrm{m}$ sections used for histological analysis.

Fig. 2 Schematic diagram illustrating the method used for geometry acquisition and creation of a specimen-specific finite element model of a representative osteochondral plug. a Diagram showing the turnstile and CCD camera used to photograph the osteochondral plug from the side. SB, subchondral bone; CC, calcified cartilage. b Photograph of the side of an osteochondral plug at one viewing angle. Red line Articular cartilage segmented using an intensity threshold. Black dots Interface of cartilage and calcified cartilage (tidemark) segmented manually. c Point cloud generated by segmented data from each viewing angle aligned to a global coordinate system. Red lines Articular surface. Blue lines Articular cartilage ascending from the articular surface. Black line Tidemark. d Surface interpolated to the points of the 3D tidemark line representing the interface between calcified cartilage and cartilage. e Wrapped and surfaced geometries of cartilage and the combined layer of subchondral bone and calcified cartilage. Green shading Articular cartilage. Red shading Subchondral bone and calcified cartilage. f Specimen-specific finite element model of the osteochondral plug. White shading Articular cartilage. Green shading Subchondral bone and calcified cartilage. 
Fig. 3 Schematic diagram illustrating the customized mechanical loading apparatus. Some structural components are not shown for clarity. The linear actuator moved the central loading column (to which the stainless steel rod and specimen were attached) in the vertical direction to apply compressive load to the specimen. LVDT, linear variable differential transformer.

Fig. 4 Experimental measurements used to calculate the Young's modulus of the combined layer of subchondral bone and calcified cartilage, and the maximum and minimum radial strains $\left(\varepsilon_{\mathrm{z}}\right.$ and $\varepsilon_{\mathrm{y}}$, respectively), (see equations (1)-(4) in the text). The $\mathrm{x}$-axis was aligned perpendicular to the articular surface and pointing above the surface; the z-axis was aligned with the maximum radial strain direction on the articular surface; and the $\mathrm{y}$-axis was orthogonal to the $\mathrm{x}$ and $\mathrm{z}$ axes. a Side view of the osteochondral plug prior to the mechanical testing. $t_{L}$ and $t_{R}$ represent the thicknesses of the combined layer of subchondral bone and calcified cartilage on the left and right edges, respectively. White line Outline of the layer of subchondral bone and calcified cartilage segmented manually. b Side view of the osteochondral plug obtained at an increment of the experimental compression. White line Outline of the deformed layer of subchondral bone and calcified cartilage segmented manually. c Image corresponding to part a showing the articular surface (viewed from the bottom) prior to the compression. White line Ellipse fitted to the outer edge of the contact area. $d_{z}$ and $d_{y}$ represent the semi-major and semi-minor diameters of the ellipse, respectively. d Image corresponding to part b showing the articular surface (viewed from the bottom) obtained at an increment of the experimental compression. White line Ellipse fitted to the outer edge of the contact area.

Fig. 5 Representative safranin-orange/fast green histology sections depicting measurement of proteoglycan (PG) thickness and OARSI grades 0-4. a Upper and lower boundaries of PG stained region. Yellow dots Upper and lower boundaries of PG region segmented manually based 
on light intensity and color. The distance between each dot on the upper boundary and the nearest dot on the lower boundary was computed. These distances were averaged and normalized by the cartilage thickness $\left(\mathrm{t}_{\text {cart }}\right)$ to yield the normalized PG thickness. b OARSI grade 0: surface intact, cartilage morphology intact. c OARSI grade 1: surface intact. d OARSI grade 2: surface discontinuity. e OARSI grade 3: vertical fissures (clefts). f OARSI grade 4: erosion.

Fig. 6 Representative picrosirius red histology section viewed under polarized light depicting the segmentation of depth-dependent zones. Yellow dots Boundaries between each zone or region segmented manually based on light intensity and color. SZ, superficial zone; IZ, intermediate zone; DZ, deep zone.

Fig. 7 Box and whisker plot of the hyperelastic material constants calculated for each OARSI grade and a linear regression line fitted to the data. The Pearson's correlation coefficient (R) and significance (p) are indicated. The red line represents the median, the edges of the box are the 25th and 75th percentiles, and the whiskers extend to the maximum and minimum of the data. The number of pooled control and pooled OA specimens for each OARSI grade are specified. Symbols appearing in the graph are as follows: $\mu$, shear modulus under infinitesimal strain associated with the neo-Hookean model; $\mathrm{C}_{10}$ and $\mathrm{C}_{20}$, constants associated with the Yeoh constitutive model.

Fig. 8 Variation of measured and simulated contact variables (contact force, average contact pressure, contact area and radial strain ratio) plotted against cartilage axial strain. The bottom panel presents the combined layer of subchondral bone and calcified cartilage $(\mathrm{SB}+\mathrm{CC})$ axial strain plotted against cartilage axial strain. Radial strain ratio was calculated as the maximum radial strain at the articular surface $\left(\varepsilon_{\mathrm{z}}\right)$ divided by the minimum radial strain at the surface $\left(\varepsilon_{\mathrm{y}}\right)$. 
$\mathrm{SB}+\mathrm{CC}$ axial strain was calculated as the maximum axial strain along the edges of the combined subchondral bone and calcified cartilage layer. Error bars indicate \pm 1 SD for the experimental data. The red lines and red shaded areas represent the means and $\pm 1 \mathrm{SD}$, respectively, for simulated results obtained from the neo-Hookean model. The black lines represent the mean for simulated results obtained from the Yeoh model. Data representing \pm 1 SD for the Yeoh model were indistinguishable from the red shaded area and are therefore not shown. The blue lines and blue shaded areas represent the means and $\pm 1 \mathrm{SD}$, respectively, for simulated results obtained by representing the $\mathrm{SB}+\mathrm{CC}$ layer as a linear-elastic material.

Fig. 9 Box and whisker plot of the RMS errors for contact force, average contact pressure, and contact area calculated for each specimen using the neo-Hookean and Yeoh constitutive models. RMS errors were calculated as a percentage of the corresponding peak values. Peak values measured for contact force, average contact pressure and contact area were $54.0 \pm 17.5 \mathrm{~N}, 6.3 \pm 2.3$ $\mathrm{MPa}$, and $8.7 \pm 1.0 \mathrm{~mm}^{2}$, respectively, for the control specimens; and $38.0 \pm 18.4 \mathrm{~N}, 4.0 \pm 2.4 \mathrm{MPa}$, and $9.8 \pm 1.8 \mathrm{~mm}^{2}$, respectively, for the OA specimens. The red line represents the median, the edges of the box are the 25th and 75th percentiles, and the whiskers extend to the maximum and minimum values of the data. ${ }^{* *} \mathrm{p}<0.001$. 
Fig. 1

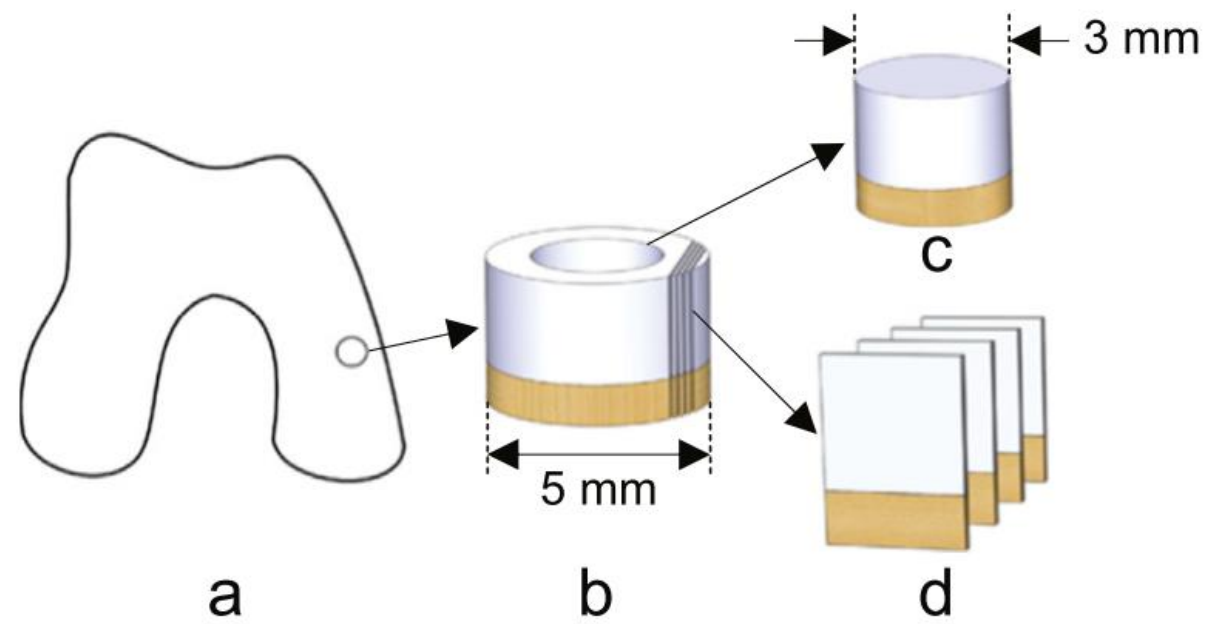


Fig. 2

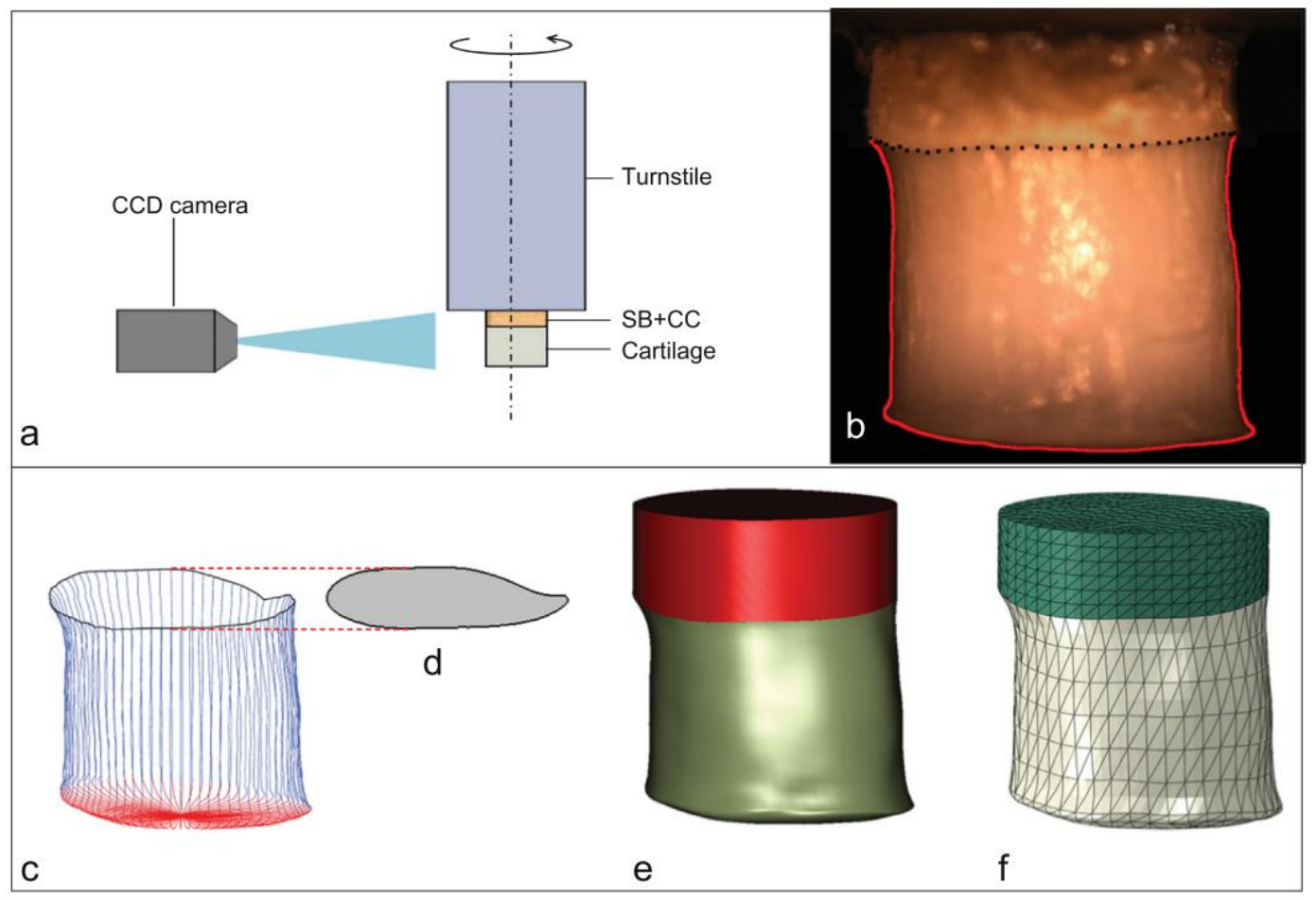


Fig. 3

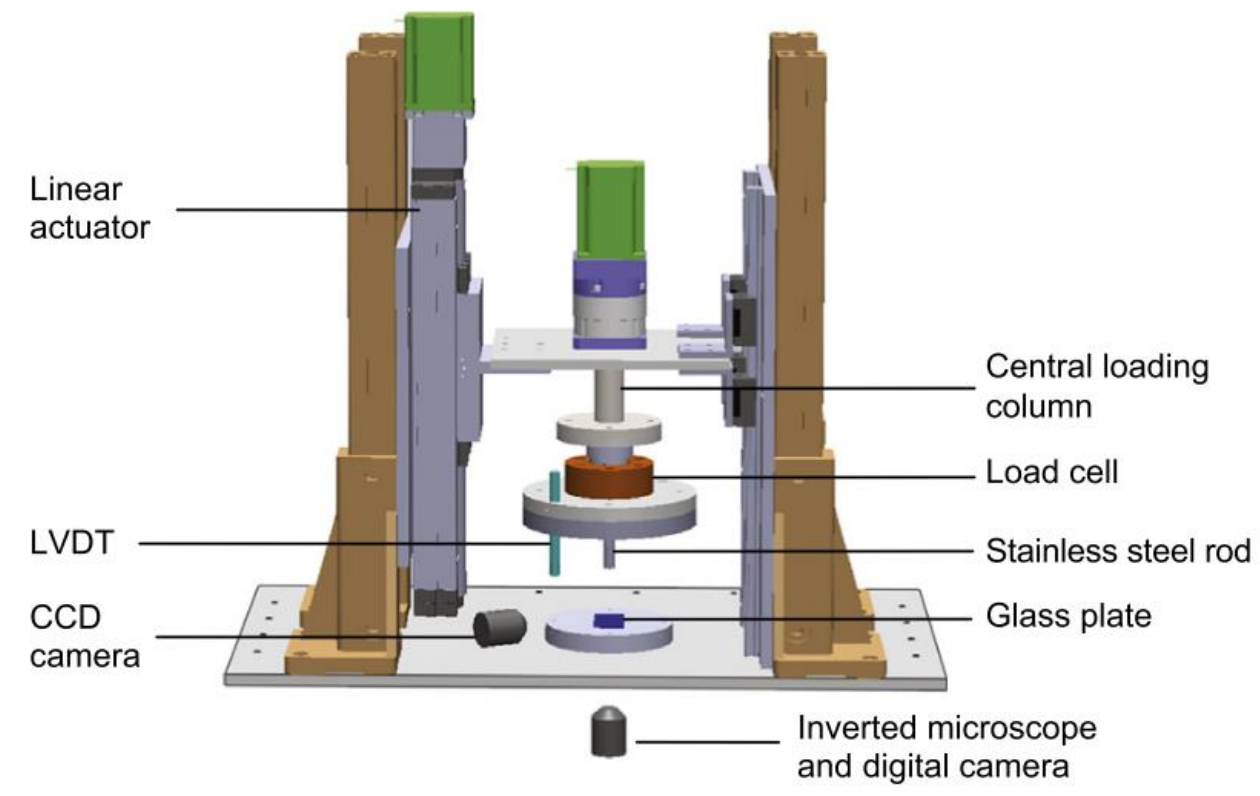


Fig. 4

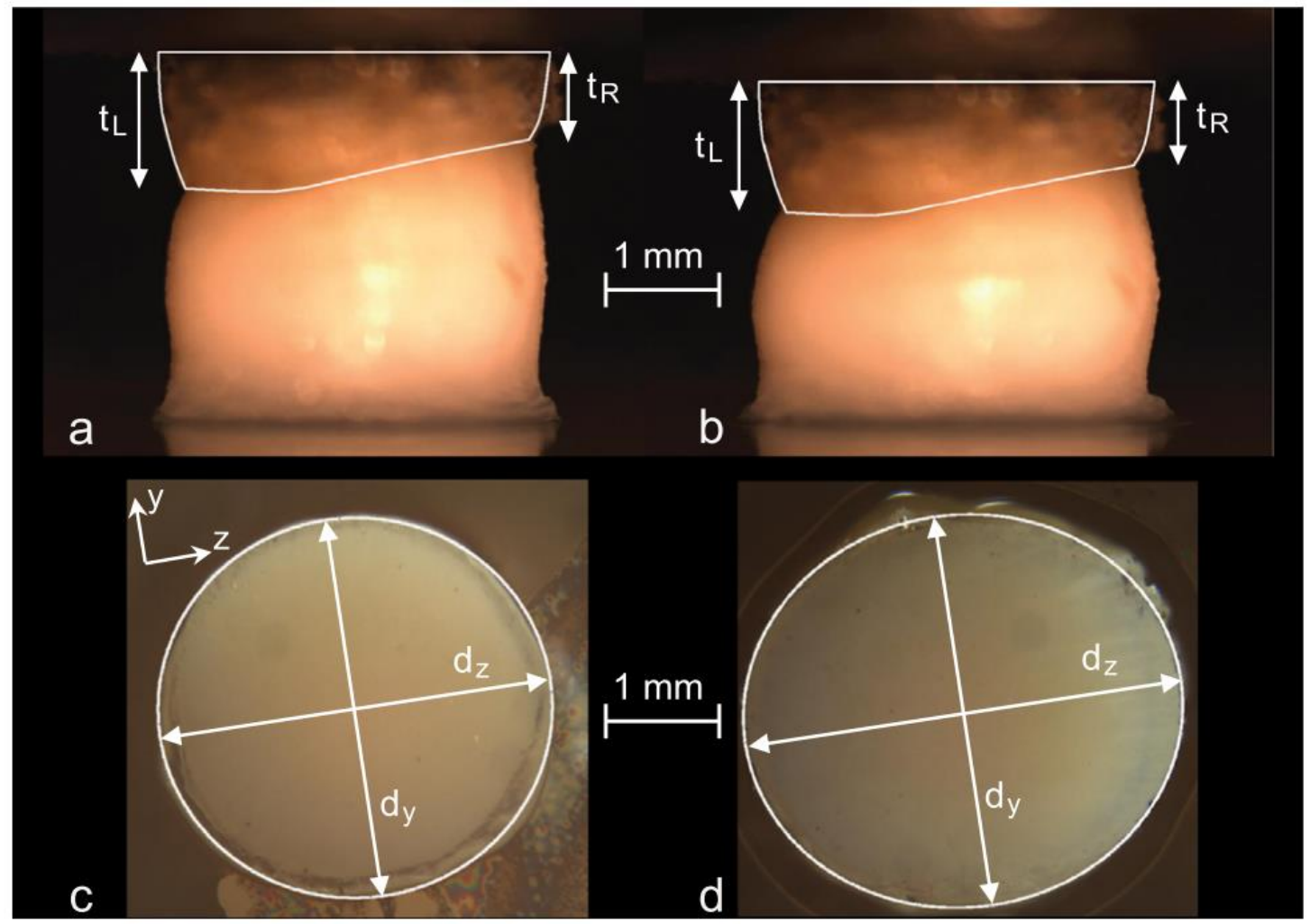


Fig. 5

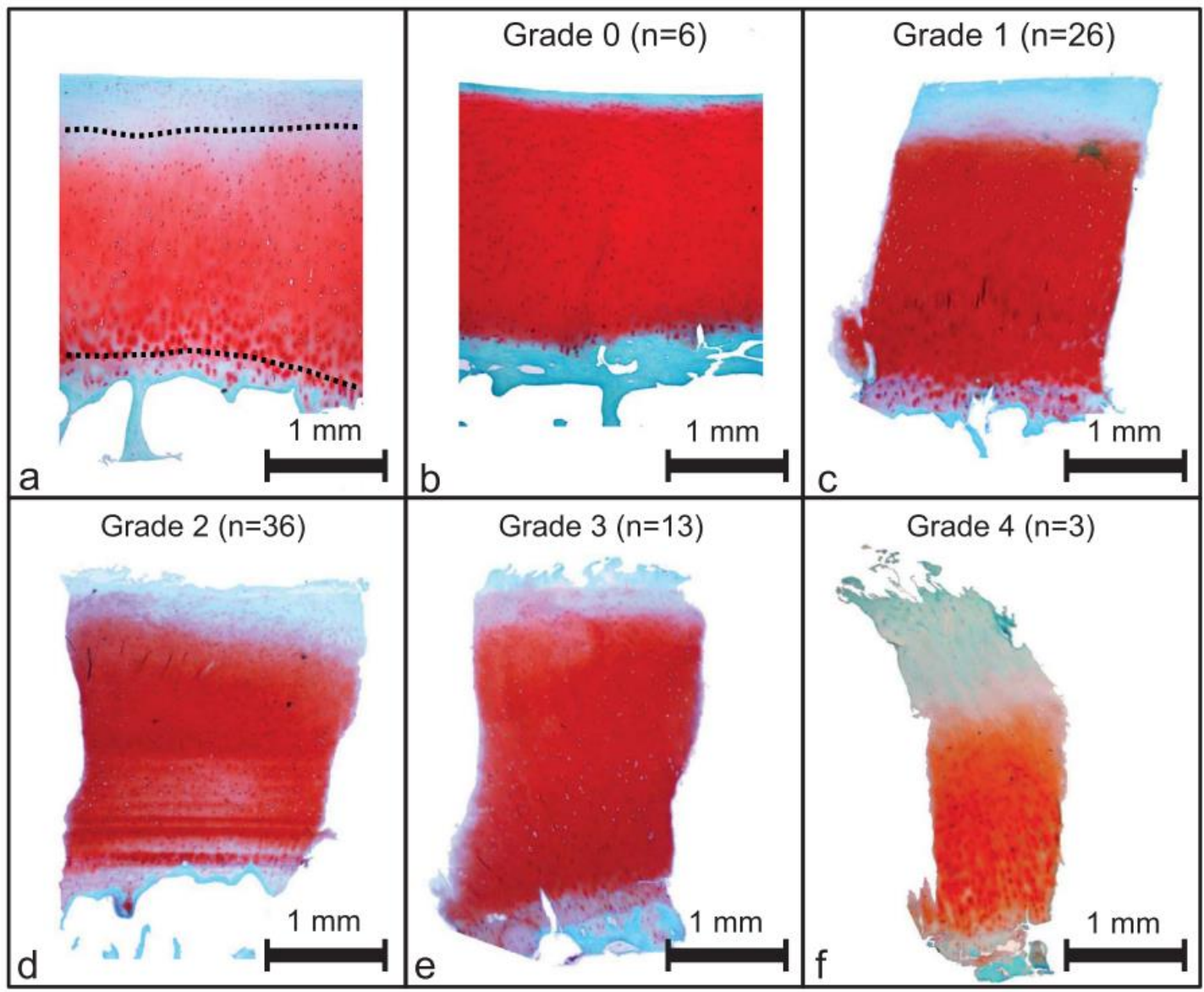


Fig. 6

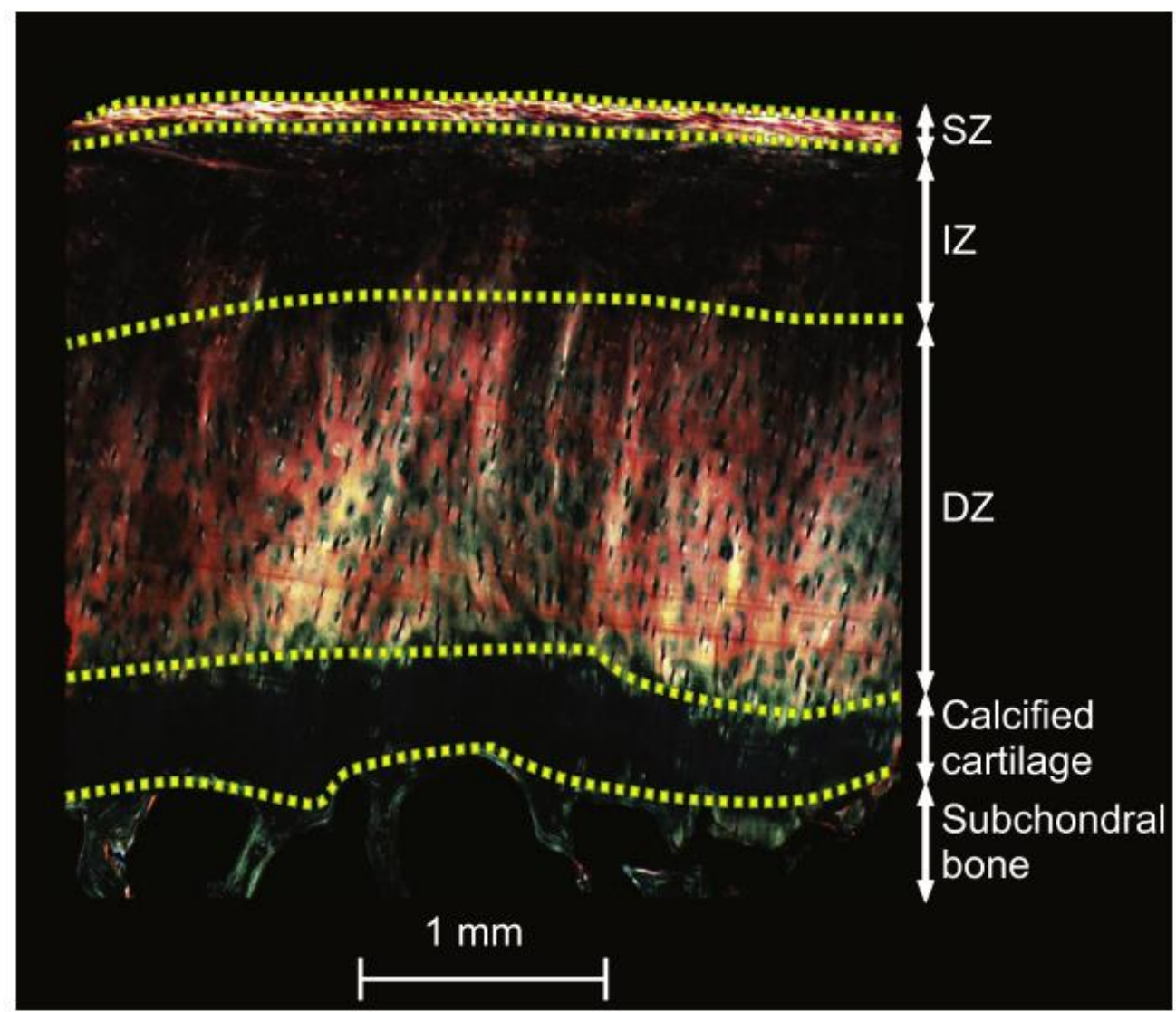


Fig. 7

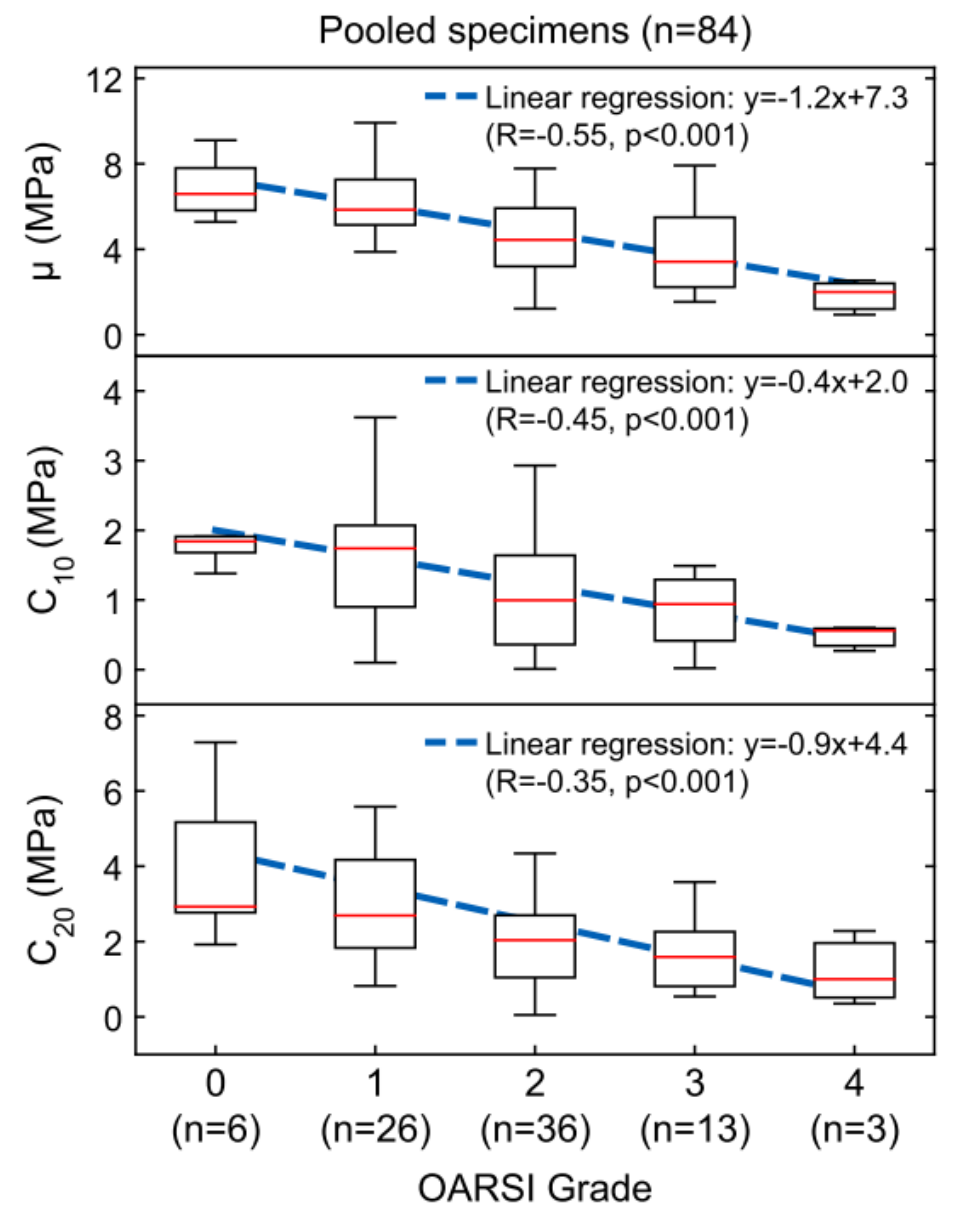


Fig. 8

Pooled normal specimens $(n=32)$

Pooled OA specimens $(n=52)$
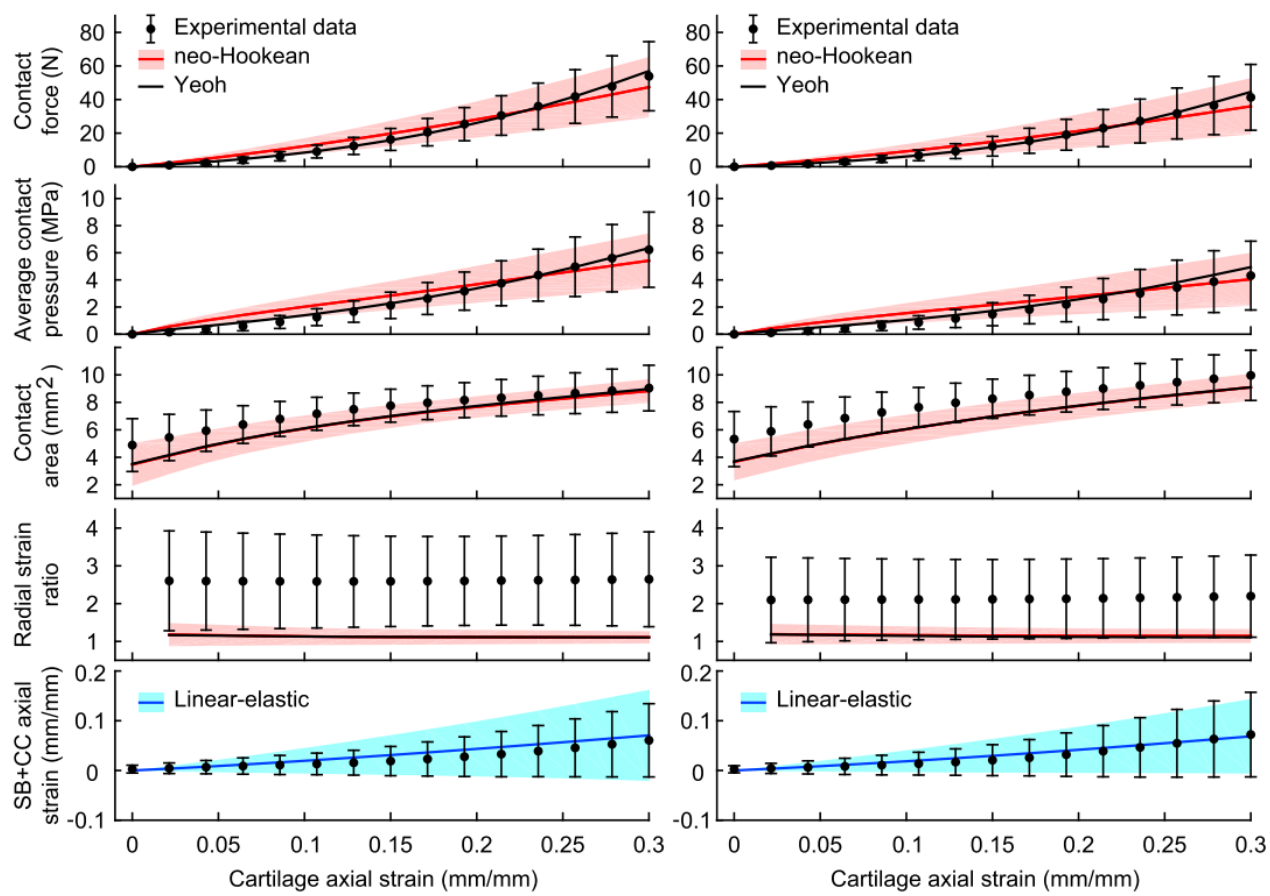
Fig. 9

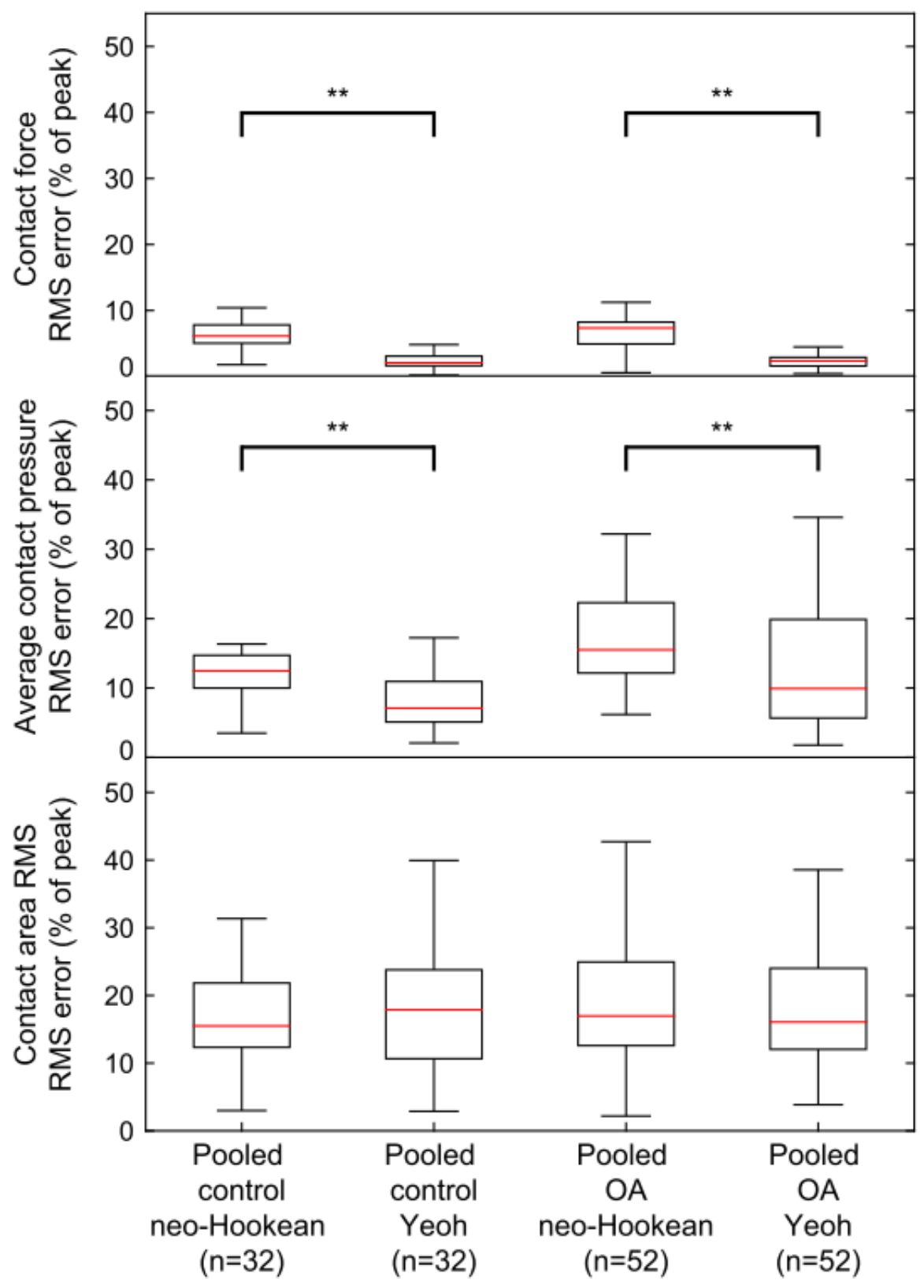




\section{University Library}

\section{- M M N E R VA A gateway to Melbourne's research publications}

Minerva Access is the Institutional Repository of The University of Melbourne

\section{Author/s:}

Robinson, DL;Kersh, ME;Walsh, NC;Ackland, DC;de Steiger, RN;Pandy, MG

Title:

Mechanical properties of normal and osteoarthritic human articular cartilage

Date:

2016-08-01

Citation:

Robinson, D. L., Kersh, M. E., Walsh, N. C., Ackland, D. C., de Steiger, R. N. \& Pandy, M.

G. (2016). Mechanical properties of normal and osteoarthritic human articular cartilage.

JOURNAL OF THE MECHANICAL BEHAVIOR OF BIOMEDICAL MATERIALS, 61, pp.96-109. https://doi.org/10.1016/j.jmbbm.2016.01.015.

Persistent Link:

http://hdl.handle.net/11343/249520 